\title{
Vulvar Sarcoma
}

National Cancer Institute

\section{Source}

National Cancer Institute. Vulvar Sarcoma. NCI Thesaurus. Code C40317.

A malignant mesenchymal neoplasm that arises from the vulva. Representative examples include childhood botryoid-type embryonal rhabdomyosarcoma, alveolar soft part sarcoma, and leiomyosarcoma. 